\title{
Effects of transport using an unmanned aerial vehicle on blood samples for cross-matching tests
}

\begin{abstract}
Objective: This study aimed to examine the effects of transport using an unmanned aerial vehicle (UAV) on blood samples for cross-matching tests in the transfusion of red blood cell solution.

Materials and methods: Two bags of red blood cell (RBC) solution (blood type, A, RBC\#1 and \#2; $280 \mathrm{~mL}$ per bag) were obtained from the Japan Red Cross Society. The RBC solution in each bag was further separated into three bags ( $93 \mathrm{~mL}$ per bag). The UAV group consisted of RBC solution with a segment tube containing the whole blood in a temperature controlled cooler box transported using an UAV. The control group consisted of RBC solution with a segment tube containing the whole blood in an in-house refrigerator that was not transported. Samples from the RBC solution were collected on day 10 (2 days before transport) and day 13 (day after transport), and lactate dehydrogenase (LD), potassium, blood sugar, and lactic acid levels were measured. After transport, cross-matching tests were performed using whole blood in the segment tubes and patient's blood (blood type, type A).
\end{abstract}

Results: We compared the biochemistry between the UAV and control group and found no significant differences, except for LD level. Although the LD level in the UAV group slightly increased compared with that in the control group, the whole blood containing the segment tubes was not hemolyzed, and cross-matching tests were performed.

Conclusion: We could fly and transport an RBC solution with segment tubes using an UAV, and this mode of transport did not affect the cross-matching tests, despite mild hemolysis in the RBC solutions.
Volume 8 Issue 5 - 2020

\author{
Michiyo Nakahara,' Miyuki Teratani,' Koki \\ Yakushiji, ${ }^{2}$ Fumiatsu Yakushiji, ${ }^{3}$ Hiroshi Fujita ${ }^{4}$ \\ 'Clinical Laboratory, Tokyo Metropolitan Bokutoh Hospital, \\ Japan \\ ${ }^{2}$ Graduate School of Media and Governance, Keio University, \\ Japan \\ ${ }^{3}$ Department of Internal Medicine, Tokyo Metropolitan Bokutoh \\ Hospital, Japan \\ ${ }^{4}$ Department of Transfusion Medicine, Tokyo Metropolitan \\ Bokutoh Hospital, Japan
}

\begin{abstract}
Correspondence: Hiroshi Fujita, MD, PhD, Director of Department of Transfusion Medicine, Tokyo Metropolitan Bokutoh Hospital, 4-23-15, Koutoubashi, Sumida-ku, Tokyo, |30-8675, Tel 8|-3-3633-6|5|, Fax 8|-3-3633-6|73, Email hiroshi_ujita@tmhp.jp
\end{abstract}

Received: August 21, 2020 | Published: September 17, 2020

Keywords: disaster, active transport refrigerator, red blood cell solution

Abbreviations: RBC solution, red blood cell solution; UAV, unmanned aerial vehicle

\section{Letter to the editor}

We first reported the transport of red blood cell (RBC) solutions using an unmanned aerial vehicle (UAV) in Japan. ${ }^{1}$ In addition, we repeated the transport of the RBC solution using an UAV in various conditions. ${ }^{2}$ In these studies, we found mild hemolysis in the transported RBC solution. ${ }^{1,2}$ We previously reported the long-distance transport of an RBC solution from Tokyo to Ogasawara Islands using an active transport refrigerator (ATR) (FUJIFILM Toyama Chemical Co. Ltd., Tokyo, Japan) by ship. ${ }^{3}$ We could safely move the RBC solutions without hemolysis. ${ }^{3}$ We also reported that transport of RBC solution by car induced hemolysis under inappropriately temperaturecontrolled and oscillated conditions. ${ }^{4}$ However, the effects of the transport using an UAV on the whole blood containing the segment tubes were neither examined nor reported.

In this letter, we show the data from the experiment performed in the Nagano Tomi Fields, where our driving UAV was examined. ${ }^{5}$ Two bags of RBC solution (blood type A, day 10) were transported in an ATR from Tokyo to Nagano by car. Using an UAV (M1000, Mazex Co. Ltd., Osaka) for $300 \mathrm{~m}$ at a maximum height of $15 \mathrm{~m}$ and an average speed of $15 \mathrm{~km} / \mathrm{h}$, the four bags of RBC solution with segment tubes in the temperature-controlled cooler box (Campers Collection, Yamazen, Corp., Tokyo, Japan) were transported. An electronic watch logger recorded the temperature each minute (KT255U, Fujita Electric Works, Ltd, Kanagawa, Japan), and a solid coolant that changed to a liquid state at approximately $2 \sim 6^{\circ} \mathrm{C}$ was used to control the temperature of the blood packs (Cold-packs Type
3, JSP Corp., Tokyo, Japan). We compared the biochemistry between the UAV and control groups and found no significant differences, except for the LD level (data not shown). Although the LD level in the UAV group slightly increased, the LD level post-filtration was extremely similar between the UAV and control groups (Table 1). In contrast, the whole blood containing the segment tubes grossly did not hemolyzed (Figure 1A \& B). As shown in Figure 1C, the supernatant of RBC solution transported by a drone revealed mild hemolysis. We first cross-matched for compatible blood from the patients with blood type A (Figure 2A, saline method; 2B, indirect antiglobulin test).

Table I Comparison of lactic dehydrogenase level of the red blood cell solution between the unmanned aerial vehicle (UAV) and control groups

\begin{tabular}{lll}
\hline & $\begin{array}{l}\text { \% of control (LD) } \\
\text { Post-transportation }\end{array}$ & $\begin{array}{l}\text { \% of control (LD) } \\
\text { Post-filtration }\end{array}$ \\
\hline RBC \#I & & \\
RBC \#I-I drone & $102 \pm 3^{*}$ & $114 \pm 3$ \\
RBC\#I-2 drone & $106 \pm I^{*}$ & $135 \pm 3$ \\
RBC\#I-3 in-house & $92 \pm 2$ & $133 \pm 6$ \\
RBC \#2 & & $112 \pm 1$ \\
RBC \#2-I drone & $166 \pm 1$ & $117 \pm 1$ \\
RBC\#2-2 drone & $194 \pm I^{*}$ & $112 \pm 1$ \\
RBC\#2-3 in-house & $169 \pm 2$ & \\
\hline
\end{tabular}

*Post-filtration implies that the RBC solution was passed through a transfusion filter (Terumo Co. Tokyo) and used at the transfusion practice

We calculated \% of control (LD) as follows:

|(LD level post-stored or post-filtered) $\div$ mean value of LD level pre-stored or pre-filtered $\times 100(\%)$

Data are expressed as group mean \pm standard deviation of the mean 
(A) Segment RBC\# 1

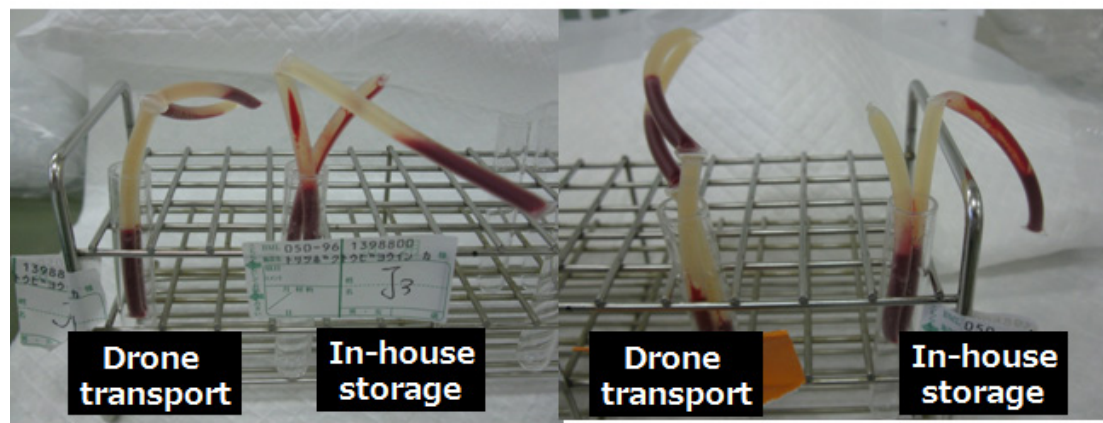

(C) Supernatant of RBC\# 1

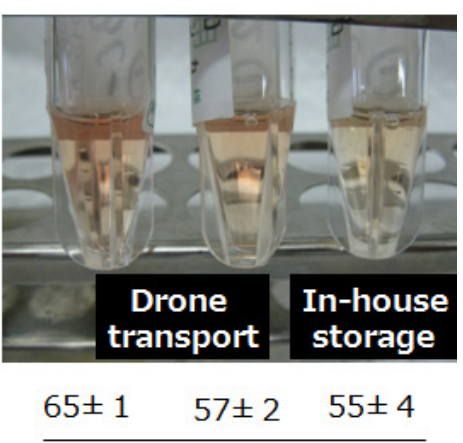

(LD (IU/L)

Figure I Whole blood in the segment tubes did not show hemolysis, and the red blood cell solution showed mild hemolysis.

The UAV (MI000, Mazex Co. Ltd., Osaka) safely transported the RBC solution with segment tubes in the Nagano Tomi Fields, where our driving UAV was examined.

(A) Gross findings of segment tubes in the RBC\#I transported by drone (right) and in-house storage (left)

(B) Gross findings of segment tubes in the RBC\#2 transported by drone (right) and in-house storage (left)

(C) Supernatant of RBC \#I

LD level (IU/L) was measured by BML Laboratories (Tokyo, Japan).

\section{(A) Saline method}

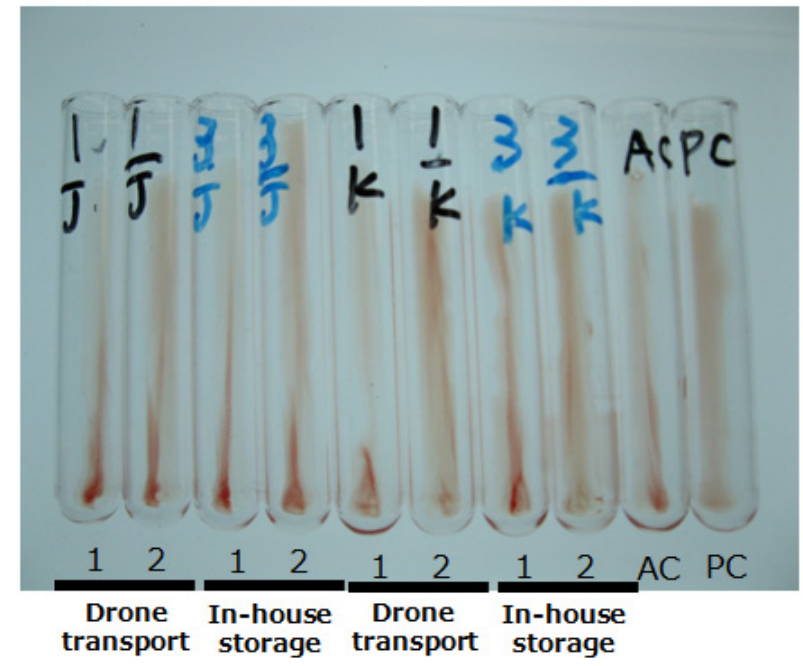

\section{(B) Indirect antiglobulin test}

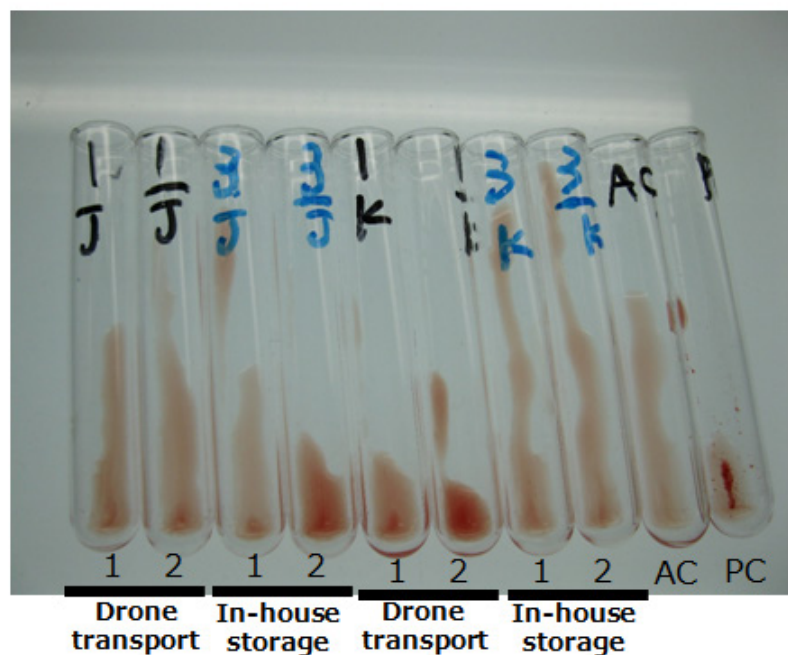

Figure 2 Results on the cross-matching tests.

We cross-matched for compatible blood from the patients with the samples in the segment tubes.

(A) Saline method

(B) Indirect antiglobulin test

Cross-matching test is a routine laboratory test for transfusion. I denotes a major test, and 2 denotes a minor test. AC, auto-control; PC, positive control

\section{Conclusion}

In conclusion, we could fly and transport an $\mathrm{RBC}$ solution with segment tubes using an UAV, and this mode of transport did not significantly affect the cross-matching tests, despite mild hemolysis in the RBC solutions (Table 1, Figure 1C). Therefore, we envisage the possibility that RBC solutions can be transported using an UAV in the event of a disaster, with the viewpoints of cross-matching tests for transfusion.

\section{Acknowledgments}

None. 


\section{Conflicts of interest}

The authors have no conflicts of interest to disclose.

\section{References}

1. Yakushiji K, Yakushiji F, Fujita H. Initial trial on the transport of blood products using an unmanned aerial vehicle (UAV) in Japan in preparation for a disaster. Hematol Transfus Int J. 2019;7(3):77-78.
2. Yakushiji F, Yakushiji K, Murata M, et al. The quality of blood is not affected by drone transport: An evidential study on the unmanned aerial vehicle conveyance of transportation material in Japan. Drones. 2020;4.

3. Igarashi T, Fujita H, Asaka H, et al. Patient rescue, and blood utilization in the Ogasawara blood rotation system. Transfusion. 2018;58(3):788-94.

4. Fujita H, Tojo Y, Mine T, et al. Temperature management of red blood cell solution transported by car for transfusion at home. Open J Blood Dis. 2020;10:37-40. 